\title{
A Stochastic Model of the Electrically Stimulated Auditory Nerve: Single-Pulse Response
}

\author{
Ian C. Bruce,* Member, IEEE, Mark W. White, Member, IEEE, Laurence S. Irlicht, Member, IEEE, \\ Stephen J. O’Leary, Scott Dynes, Eric Javel, and Graeme M. Clark
}

\begin{abstract}
Most models of neural response to electrical stimulation, such as the Hodgkin-Huxley equations, are deterministic, despite significant physiological evidence for the existence of stochastic activity. For instance, the range of discharge probabilities measured in response to single electrical pulses cannot be explained at all by deterministic models. Furthermore, there is growing evidence that the stochastic component of auditory nerve response to electrical stimulation may be fundamental to functionally significant physiological and psychophysical phenomena. In this paper we present a simple and computationally efficient stochastic model of single-fiber response to single biphasic electrical pulses, based on a deterministic threshold model of action potential generation. Comparisons with physiological data from cat auditory nerve fibers are made, and it is shown that the stochastic model predicts discharge probabilities measured in response to single biphasic pulses more accurately than does the equivalent deterministic model. In addition, physiological data show an increase in stochastic activity with increasing pulse width of anodic/cathodic biphasic pulses, a phenomenon not present for monophasic stimuli. These and other data from the auditory nerve are then used to develop a population model of the total auditory nerve, where each fiber is described by the single-fiber model.
\end{abstract}

Index Terms - Auditory nerve, cochlear implant, functional electrical stimulation, population response, sensory prosthesis, single-pulse response, stochastic threshold model.

\section{INTRODUCTION}

A CCURATE models of auditory nerve (AN) response to electrical stimulation may help improve our under-

Manuscript received October 20, 1997; revised January 5, 1999. This work was supported by the Human Communication Research Centre, The University of Melbourne, the Bionic Ear Institute, the CRC for Cochlear Implants, Speech and Hearing Research, the National Institutes of Health under Grant DC00361, and the Cochlear Implant Research Laboratory, Massachusetts Eye and Ear Infirmary. Asterisk indicates corresponding author.

*I. C. Bruce was with the Bionic Ear Institute and the Department of Otolaryngology, The University of Melbourne, East Melbourne VIC 3002, Australia. He is now with the Department of Biomedical Engineering, 505 Traylor Bldg., Johns Hopkins University, 720 Rutland Ave, Baltimore, MD 21205 USA (e-mail: ibruce@bme.jhu.edu).

M. W. White is with Department of Electrical and Computer Engineering, North Carolina State University, Raleigh, NC 27695 USA.

L. S. Irlicht was with the Bionic Ear Institute and the Department of Otolaryngology, The University of Melbourne, East Melbourne, VIC 3002, Australia. He is now with County Investment Management, Melbourne VIC 3000, Australia.

S. J. O'Leary and G. M. Clark are with the Bionic Ear Institute and the Department of Otolaryngology, The University of Melbourne, East Melbourne VIC 3002, Australia.

S. Dynes is with the Eaton-Peabody Laboratory, Massachusetts Eye and Ear Infirmary, Boston, MA 02114 USA.

E. Javel is with the Department of Otolaryngology, University of Minnesota, Minneapolis, MN 55455 USA.

Publisher Item Identifier S 0018-9294(99)03977-4. standing of how auditory percepts are produced by cochlear implants and prompt the development of new stimulation strategies that better code speech for implant users [1], [2]. Historically, neural response to electrical stimulation has been modeled via deterministic descriptions (exceptions among cochlear implant groups include [2]-[8]; and in other fields of neuroscience [9], [10]). Deterministic descriptions specialized to AN anatomy and physiology include the Colombo and Parkins [11] and Frijns et al. [12] models. However, a small amount of stochastic activity was present in the squid giant axon [13] and frog node [14], [15] data on which many deterministic models are based [16]. Furthermore, potentially significant variance has been measured in the responses of fibers to single-current pulses [7], [17]-[19] and pulse trains [8], [20], [21], which cannot be explained by deterministic models.

The amount of variance is dependent on the stimulus intensity, and it has generally been assumed that cochlear implants operate at stimulus intensities that produce extremely high discharge probabilities (very close or equal to one) in AN fibers, resulting in very little variance. In contrast, preliminary evidence is emerging that cochlear implants may be operating at intensities that produce low to moderate discharge probabilities, resulting in significant variance [22]-[24]. If this is correct, then there are important consequences for cochlear implants. For example, i) both temporal and spatial aspects of speech coding would be impacted [21]-[24], and ii) the survival of AN fibers subject to high stimulation rates is likely to be strongly affected [25], [26].

Verveen and Derksen showed that the variance in response to single-current pulses can be attributed to random fluctuations in the voltage across the membrane of myelinated fibers at the nodes of Ranvier [27]. They recorded membrane potentials in vitro from the sciatic nerve of the frog, Rana temporaria. Fig. 1 shows a number of their membrane-potential traces at $-90 \mathrm{mV}$, Fig. 1(a), and at a range of depolarized and hyperpolarized potentials, Fig. 1(b) - resting potential is $\sim-70 \mathrm{mV}$ [28].

Verveen and Derksen characterized these fluctuations as having a Gaussian amplitude distribution for membrane potentials between -70 and $0 \mathrm{mV}$ [29] and a $1 / f$ frequency spectrum [27]. Three of their observations are worth highlighting. First, the Gaussian fit is accurate at least between the probabilities of 0.001 and 0.999 , if not for even finer probabilities. Second, Verveen and Derksen showed that the variance of the fluctuations increases with depolarization, as seen in the upper traces of Fig. 1(b). Third, they showed that 


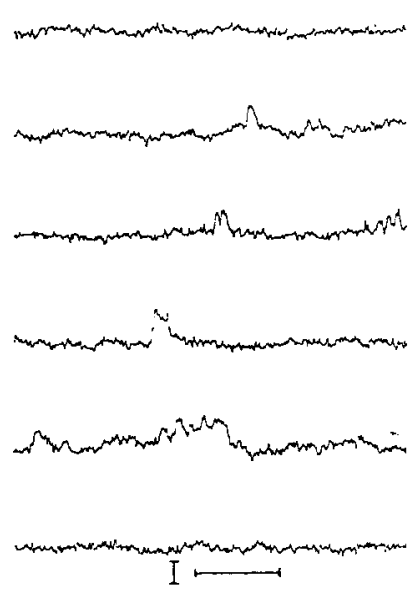

(a)

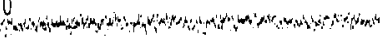

$-17$

$-42$

.59


(b)
Fig. 1. Membrane-potential traces from [27] showing fluctuations in nerve fiber transmembrane potentials at the nodes of Ranvier. (a) Membrane potential traces at $-90 \mathrm{mV}$. Horizontal scale: $10 \mathrm{~ms}$; vertical scale: $1 \mathrm{mV}$. (Reprinted with permission from [27, Fig. 20] (c) 1968 IEEE.) (b) Membrane potential traces at different levels of membrane potential (given in $\mathrm{mV}$ above each trace). Horizontal scale: $1 \mathrm{~ms}$; vertical scale: $5 \mathrm{mV}$. (Reprinted with permission from [27, Fig. 19] (C) 1968 IEEE.)

some nonstationary noise activity (as seen in the middle traces of Fig. 1(a) and in the lower traces of Fig. 1(b), increasing both in frequency of occurrence and in amplitude with increasing hyperpolarization) results in a positively skewed amplitude distribution for membrane potentials below $-70 \mathrm{mV}$, the skew increasing with increasing hyperpolarization.

Membrane potential fluctuations in squid axons are considered to be of negligible magnitude [16]. However, Verveen reported that the magnitude of the membrane potential fluctuations appears to be negatively correlated with the diameter of the nerve fiber [30]. Mammalian AN fibers have a mean diameter of $\sim 2-4 \mu \mathrm{m}$ [31], [32] and even smaller diameters $(0.1-0.7 \mu \mathrm{m})$ at the nodes of Ranvier in the myelinated portion of the peripheral dendrite, proximal to the habenula [33]. This is two to three orders of magnitude smaller than the mean diameter of squid giant axons ( $\sim 500 \mu \mathrm{m}$ [34]), which indicates why membrane potential fluctuations may be of significant magnitude in mammalian AN fibers.

Further physiological [28], [35] and modeling [6] studies suggest that these membrane fluctuations are likely to be caused by random transitions in the state of single ionic channels at the nodes of Ranvier. This is consistent with the magnitude of the fluctuations being negatively correlated with the diameter of the axon, since the larger the diameter of the axon, the more single ionic channels there will be at each node. The more channels, the less significant the fluctuations of any one channel will be when compared to the total ionic conductance. Indeed, introduction of the correct single-channel conductance statistics to models such as the Hodgkin-Huxley equations has produced the correct prediction of membrane potential fluctuations and the resulting response to single pulses and pulse trains [6], [9].

However, detailed stochastic descriptions of AN response to electrical stimulation based on Hodgkin-Huxley-type models [4], [6], [7] are too computationally intensive to permit simulation of a large number of neurons over hundreds of milliseconds, which may be required for the simulation to be psychophysically relevant. An alternative statistical approach is to apply a point process description of neural response to electrically elicited responses [2], [8]. This description is more computationally efficient, but since it is entirely phenomenological it is not easily related to stimulus and neural parameters. Furthermore, i) it is not clear how this model specializes to an equivalent deterministic model, and ii) the point process description is not easily generalized to a population of fibers.

In this paper, we present an alternative model of $\mathrm{AN}$ response to electrical stimulation, following the conceptual approach of [3], [5], and [27]. Based on the Hill threshold model [36], our model includes a number of significant components of action potential generation, including membrane noise as characterized by Verveen and colleagues. Threshold models are much simpler conceptually and are more computationally efficient than Hodgkin-Huxley-type models and provide a good approximation to such models [37]. Additionally, our model can be fitted easily to the statistics of AN parameters collected from physiological studies. It is, therefore, suitable for the calculation of large-scale population responses. Such responses are required for the investigation of sound coding in ensembles of nerve fibers, for the explanation or prediction of psychophysical results, and for the development of speech processing strategies for cochlear implants. Furthermore, many aspects of the stimulus and neural properties are common to functional electrical stimulation of other sensory and motor systems. Consequently, the model developed here may be useful for other prosthetic devices.

In Section II, we describe the physiological methods. In Section III we present the model of AN response to single biphasic electrical pulses and investigate response properties of the model. In particular, we compare the behavior of the model with and without the stochastic component. In Section IV, we demonstrate the model's suitability for describing large-scale population responses by developing a model of total AN response. We show how the parameters of an arbitrary number of $\mathrm{AN}$ neurons can be fitted to the statistics of AN data collected from physiological studies and how the output of the resulting total AN model varies with stimulus and model parameters. Finally, in Section $\mathrm{V}$ we discuss further physiological and modeling studies that would help in refining and extending the model and some implications of the stochastic behavior of AN response for cochlear implant research.

\section{METHODS}

The first set of physiological data presented in this paper are single-fiber cat AN data from [38]. Adult cats with normal hearing and no evidence of external or middle ear disease were anesthetized with sodium pentobarbital $(40 \mathrm{mg} / \mathrm{kg})$ and mounted in a rigid headholder. Supplemental anesthesia was given as necessary throughout the experiment. Core temperature was held at $37^{\circ} \mathrm{C}$ using a DC heating pad.

After the bulla was opened and the round window membrane reflected, a custom-built intracochlear electrode array 
was inserted into the scala tympani for a distance of approximately $6 \mathrm{~mm}$. The electrode array possessed 12 circumferential platinum bands, each having a width of $0.3 \mathrm{~mm}$. The bands were positioned on a silicone rubber carrier at $0.75-\mathrm{mm}$ centers. Once the electrode array had been installed, cyanoacrylate cement and polyester mesh were used to fix it in position.

The procedures used to expose the AN and record from single fibers were the same as those described in [39]. Electrical stimuli were biphasic charge-balanced current pulses delivered by an optically isolated current source. Pulse width (phase duration), amplitude, and repetition rate could be set under computer control. The data presented here were obtained using the most apical electrode band as the current source and the adjacent band as the current sink.

To asses the hearing status of the cat during the experiment, acoustic stimuli were synthesized digitally and presented by a condenser microphone through a calibrated distortioncompensating network and custom-made hollow speculum. Following implantation, AN fibers initially exhibited normalappearing spontaneous activity and acoustic responses. However, spontaneous activity and acoustic responses typically deteriorated as experiments proceeded past the first day and were gradually lost as experiments proceeded past the second day [38].

In this paper we present a previously unpublished analysis of the electrically elicited data set, in which we estimate the discharge probability in response to single biphasic current pulses. The discharge probability for a single biphasic pulse is estimated from the fraction of trials in which an action potential is generated in response to the first pulse of a pulse train. Because the first pulse of each pulse-train occurs at least $50 \mathrm{~ms}$ after the end of the preceding pulse-train, the response to the first pulse should closely approximate the response to a single pulse in isolation [21]. For each neuron we pool data of the same stimulus intensity and pulse width across trains of different pulse rates.

The method for suppressing the stimulus artifact meant that little data was collected for stimuli with pulse widths above $400 \mu \mathrm{s} / \mathrm{phase}$ and none above $600 \mu \mathrm{s} / \mathrm{phase}$. Pulse width has a significant effect in a range of psychophysical measures, and we would, therefore, like the model to be accurate over a greater range of pulse widths. Thus, we also present a post hoc analysis of previously unpublished data collected by Dynes from single AN fibers of cats, where a pair of closely spaced micropipettes were used in differential-like recording to produce a high signal-to-artifact ratio even at long pulse widths. Due to the time and difficultly involved in collecting sufficient data over the whole dynamic range of a fiber, it was not possible to collect data with an exhaustive set of stimulus parameters. It was, therefore, decided to use only monopolar stimulation, such that fibers should respond preferably to the cathodic phase [36], [40]. A cathodic "search" pulse was used to find AN fibers responsive to cathodic stimulation, and then responses to anodic/cathodic biphasic pulses were recorded-it was believed from Verveen and colleagues' data that i) the anodic phase may act as a "conditioner" stimulus [7] that would affect the fiber's response to the cathodic phase, and ii) such effects may be dependent on the pulse width.

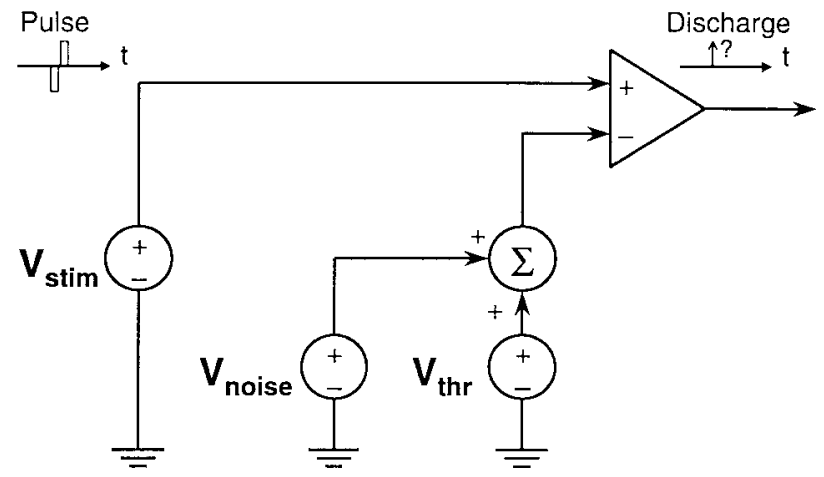

Fig. 2. Stochastic model of single-pulse response. See text for explanation of circuit components.

For data collected by Dynes, animal preparation and techniques to record from single AN fibers are essentially as described in Kiang et al. [41]. In brief, adult cats were deafened with kanamycin and ethacrynic acid and injected peritoneally with diallyl barbituric acid in urethane solution (dosage $75 \mathrm{mg} / \mathrm{kg}$ weight).

Electrical stimulation was provided through a single Tefloncoated platinum wire (0.005 in wire diameter). The apical tip of platinum wire electrodes passed about $4-5 \mathrm{~mm}$ through the round window. In all cases, the indifferent electrode was an 18-gauge needle placed in the ipsilateral forelimb.

The electrical search stimulus was a train of $100-\mu$ s cathodic pulses presented at a rate of 10/s. The search stimulus amplitude was varied but was typically $15 \mathrm{~dB}$ below $1 \mathrm{~mA}$. Single fibers were detected by monitoring the neural potentials on an oscilloscope. The experimental stimuli consisted of symmetric anodic/cathodic biphasic pulses of durations between 100 and $5000 \mu \mathrm{s} /$ phase, presented at a rate such that there was at least an $80-\mathrm{ms}$ interval between stimuli. The estimate of discharge probability for each stimulus intensity was based on 100 stimulus presentations.

\section{Single-Pulse ResPonse}

We define a single pulse as any pulse whose response properties are not dependent on any previous pulses. The model will, therefore, be valid for any single pulse presented in isolation, for the first pulse in a pulse train, and for all pulses in a pulse train of sufficiently low pulse rate. Interpulse effects will be considered in [21], where we use the model of single-pulse response as the basis for a model of pulse-train response.

\section{A. Model of Single-Pulse Response}

Fig. 2 shows an electrical circuit representation of our stochastic single-pulse model. This model is Hill's deterministic threshold model of action potential generation [36], extended by inclusion of a Gaussian noise source.

Hill's model utilizes two "potentials," which he labels the "local potential" and the "threshold potential." We use $V_{\text {stim }}$, the "stimulus potential," to refer to Hill's "local potential" and $V_{\text {thr }}$ to refer to the threshold potential. $V_{\text {stim }}$ responds to the injection of electrical current with a process Hill calls 
"excitation." When the stimulus potential $V_{\text {stim }}$ crosses the threshold potential $V_{\mathrm{thr}}$, an action potential is generated. To model the membrane potential fluctuations characterized by Verveen, we add to $V_{\text {thr }}$ a noise potential $V_{\text {noise, }}$, which has a Gaussian amplitude distribution and a $1 / f$ spectrum.

In order to increase the ease and efficiency with which we can calculate whether or not an action potential occurs in response to a pulse, we make a series of approximations. Note that as a consequence of these approximations, the model's prediction of the exact latency of the response is degraded.

1) For biphasic current pulses, discharges may occur in response to the onset and offset of both the cathodic and the anodic phases. However, the thresholds are lower for the onset of the cathodic phase [36], [40] and we, therefore, approximate the response to biphasic pulses by the response to the onset of the cathodic phases. Furthermore, inner hair cell loss is assumed to be near complete, such that extremely little spontaneous activity exists ${ }^{1}$ [42], and consequently discharges are very unlikely to occur between pulses. We, therefore, consider that discharges may only occur in response to the cathodic phase of a biphasic current pulse and may not occur between pulses. We refer to a discharge in response to the cathodic phase of any biphasic pulse $n$ as having occurred during pulse $n$.

2) We ignore any change in $V_{\text {thr }}$ with the stimulating current (e.g., "accommodation" [36]), i.e., $V_{\text {thr }}=V_{\text {thr }}(n)$, a constant value, for the duration of the cathodic phase of pulse $n$. We also approximate $V_{\text {stim }}$ by its asymptotic value, i.e., $V_{\text {stim }}$ is considered to increase instantaneously in response to a current pulse and remain at a constant value $V_{\text {stim }}(n)$ for the duration of the cathodic phase of pulse $n$.

3) Accurate simulation of noise with a $1 / f$ frequency spectrum is quite laborious. However, the $1 / f$ shape of the spectrum is such that the low-frequency content of the noise outweighs the high-frequency content to such an extent that $V_{\text {noise }}$ changes slowly enough that it may be considered to remain constant for the duration of a short current pulse, i.e., we consider $V_{\text {noise }}$ to have a constant value $V_{\text {noise }}(n)$ for the duration of pulse $n$. This noise is normally distributed with a mean of zero and variance $\sigma^{2}$. Note that this approximation will decrease in accuracy with increasing pulse width.

Given these approximations, Monte Carlo simulation of the model is very simple. A normally distributed (mean zero, variance $\sigma^{2}$ ) pseudorandom number is generated to give a value for $V_{\text {noise }}(n)$. If $V_{\text {stim }}(n)$ is less than $V_{\text {thr }}(n)+V_{\text {noise }}(n)$, then no discharge is said to have occurred; if it is equal or greater than $V_{\text {thr }}(n)+V_{\text {noise }}(n)$, then a discharge is said to have occurred. Unfortunately this method is computationally laborious, because the stochastic version of the model requires many simulation iterations to accurately describe the distribution of responses.

\footnotetext{
${ }^{1}$ This is supported by the data presented in Section III.B — extrapolation of these data indicates very low levels of spontaneous activity.
}

However, the Monte Carlo simulation described above is in effect a Bernoulli process [43], where a discharge in response to a pulse is considered to be a success and no discharge a failure. This permits the development of an analytical expression for the discharge probability in response to any single pulse $n$.

We define the output of this model as a Bernoulli random variable $p(n)$ that is dependent on the parameters of pulse $n$ and the parameters of the neural model.

$$
p(n) \triangleq \operatorname{Pr}\{\text { discharge during pulse } n\} .
$$

For the deterministic model, i.e., for $\sigma$ of $V_{\text {noise }}(n)=0$, only two possible values of $p(n)$ exist, zero and one. If the stimulus intensity is great enough to cause $V_{\text {stim }}(n)$ to rise to $V_{\text {thr }}(n)$ or above, an action potential will always result. If the stimulus intensity is less than this critical value, no action potential will ever result. That is

$$
p(n)= \begin{cases}0, & \text { for } V_{\text {stim }}(n)<V_{\text {thr }}(n) \\ 1, & \text { for } V_{\text {stim }}(n) \geq V_{\text {thr }}(n) .\end{cases}
$$

The discharge probability versus stimulus intensity relationship can be referred to as an input/output (I/O) function.

For the stochastic model, i.e., for a nonzero $\sigma$ of $V_{\text {noise }}(n)$, the $\mathrm{I} / \mathrm{O}$ function is

$$
p(n)=\frac{1}{2}\left(1+\operatorname{erf}\left(\frac{V_{\text {stim }}(n)-V_{\text {thr }}(n)}{\sqrt{2} \sigma}\right)\right) .
$$

The function $p(n)$ is dependent on $V_{\text {stim }}(n)$ with two independent variables: $V_{\text {thr }}(n)$ and $\sigma$. As with the deterministic model, we refer to $V_{\text {thr }}(n)$ as the "threshold." From (3), this definition of threshold corresponds to the stimulus intensity that produces a discharge probability of 0.5 and is consistent with Verveen's convention for defining threshold.

Verveen referred to the second variable $\sigma$ in a normalized form, which he labeled the "relative spread" (RS) [17], [18]

$$
\mathrm{RS} \triangleq \frac{\sigma}{\text { threshold }} .
$$

The greater the value of RS, the shallower the slope of the I/O function [see Fig. 4(a) and (b)].

A full mathematical derivation of (2) and (3) is provided in [23, Ch. 5]. Predictions of single-fiber response can be computed simply and efficiently using these analytical descriptions, and multiple iterations are not required. Predictions of the physiological single-pulse data by the analytical expressions are presented in the next section.

\section{B. Model Predictions of Single-Pulse}

The integrated-Gaussian (error function) description of single-pulse discharge probability given by (3) is consistent with both physiological results [7], [17]-[19] and modeling studies [6], [9] investigating the response of nerve to monophasic pulses. However, we wish to investigate how accurately this description predicts data from the cat $\mathrm{AN}$ in response to biphasic pulses.

Fig. 3 shows the stochastic and deterministic model leastsquares fit to [38, Neuron 3-21]. For the stochastic model the 


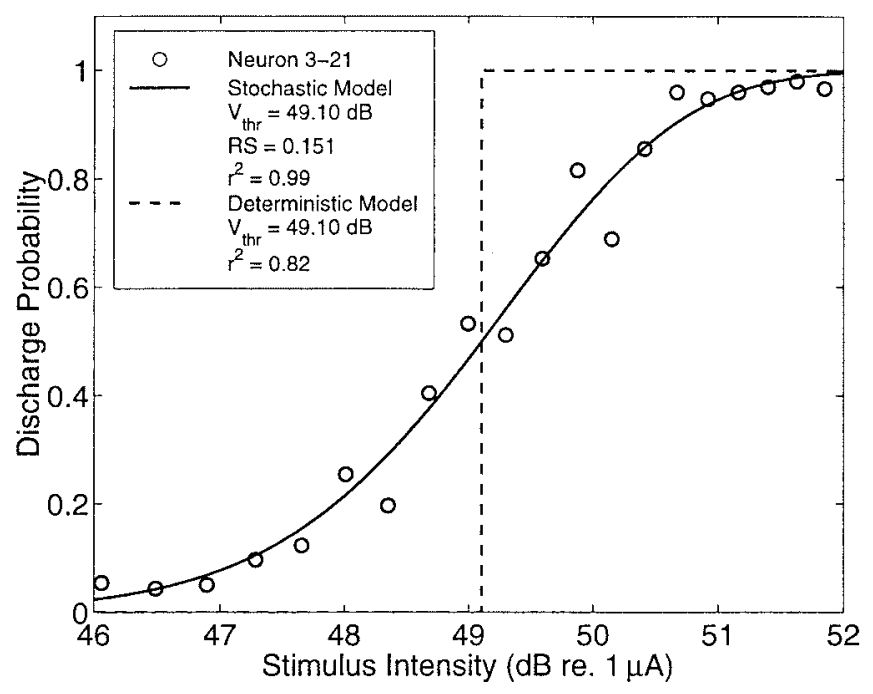

Fig. 3. Stochastic (solid line) and deterministic (dashed line) model fits to discharge probability data (o) [38, Neuron 3-21] for a single biphasic pulse of duration $100 \mu \mathrm{s} /$ phase.

variables being fitted are $V_{\text {thr }}$ and RS-the values of these for Neuron 3-21 are given in the figure legend, as is the correlation coefficient. The deterministic model fit is achieved by setting the noise term to zero, producing a step function at the $V_{\text {thr }}$ of the stochastic model fit. The correlation coefficient for the deterministic model is also given in the figure legend.

Note:

1) The 6-dB width of the horizontal scale is chosen to correspond to typical behavioral dynamic ranges of cochlear implant users in response to a single pulse [44].

2) The step-function prediction is not due to the simplicity of Hill's model-because of the level detector involved in spike generation, even the most complex deterministic models are only able to provide a step-function fit to these data.

The step-function fit is a relatively poor representation of the data, and the practical implications of fitting a step-function are significant. Information that is encoded in the discharge probability will not be accurately described by the step function. For example, the step-function can only code intensity levels as being above or below some "threshold" value, whereas the physiological data indicate that the discharge probability in a single AN fiber could be used to code intensity levels over some 3-dB to 4-dB range. Typical behavioral dynamic ranges in cochlear implant users in response to single short-duration pulses are 6-10 dB [44], and the fiber's actual I/O function covers at least half of that range. These practical implications, along with the difference in correlation coefficients, suggest that the stochastic model provides a much better description of the I/O function with the addition of only one extra parameter, RS.

Further examples of integrated-Gaussian function leastsquares fits to cat data from [38] are given in Fig. 4(a) and (b) for single biphasic pulses of durations $200 \mu \mathrm{s} /$ phase and $400 \mu \mathrm{s} /$ phase, respectively. Again, for the stochastic model the variables being fitted are $V_{\text {thr }}$ and RS, and the values of these for the four neurons are given in the figure legends, as are the correlation coefficients. Note that the abscissa is stimulus

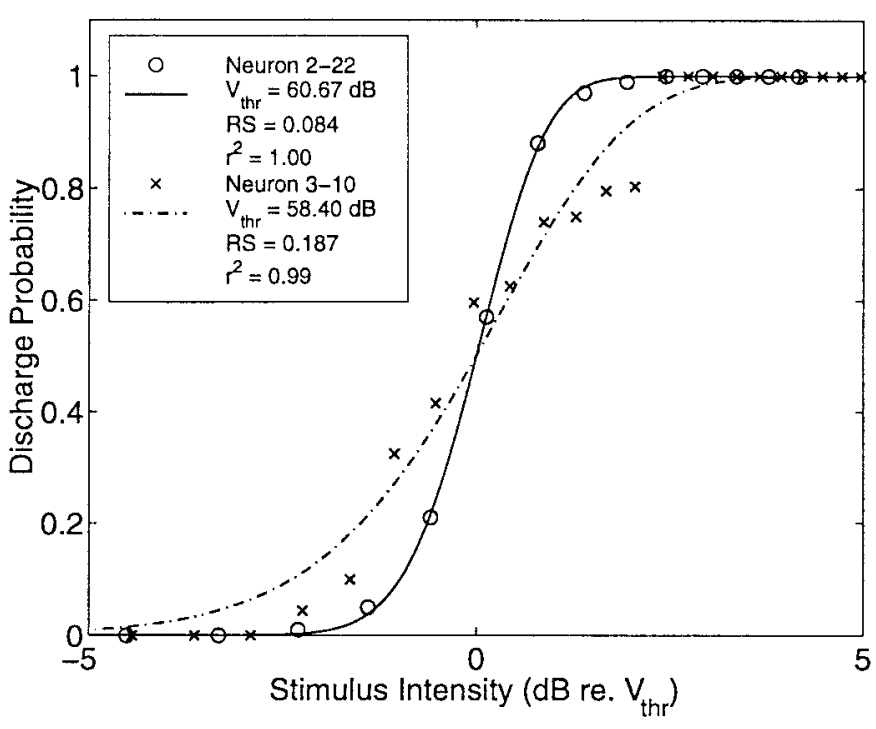

(a)

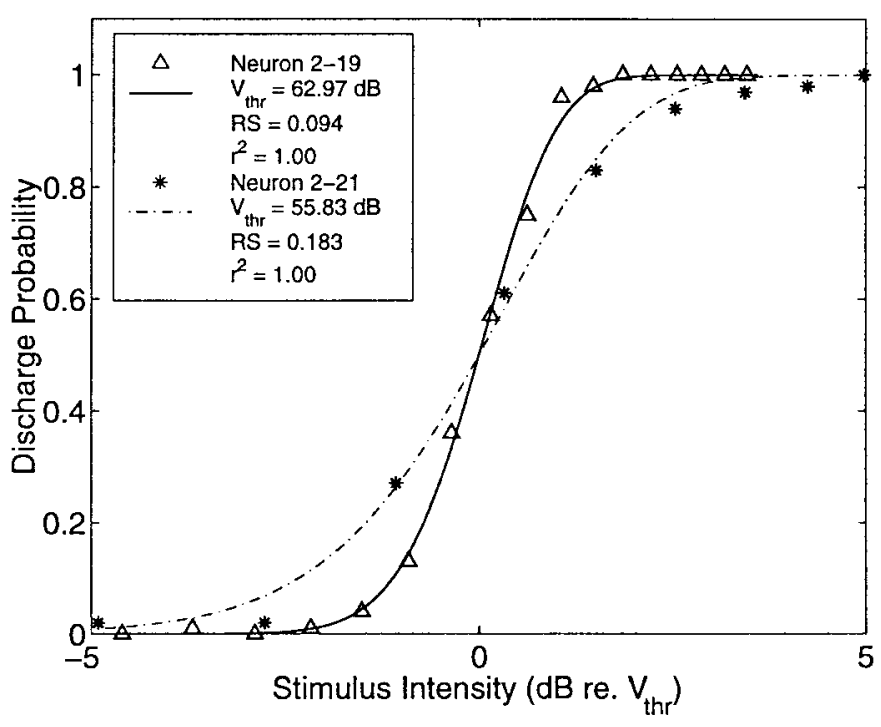

(b)

Fig. 4. Fits to fiber I/O functions from [38] for a single pulse. (a) 200 us/phase and (b) 400 us/phase. Note that the abscissa is stimulus intensity relative to the fiber's threshold.

intensity relative to the fiber's threshold, to highlight that RS values can vary quite widely for a particular phase duration. See Section IV-A for further analysis of the distribution of RS values.

\section{Total Auditory Nerve Response}

In order to model the response of a population of AN fibers we need to determine the model parameters for each neuron in the population, as well as the intensity of the excitatory current at the initial site of action potential generation in each neuron.

To demonstrate how this may be done, we develop a population model for the total AN following the approach used in [45], but using the model of single-fiber response presented in this paper. This is achieved by fitting the model parameters to the AN statistics from cat data and by using a simple model of current spread. 




Fig. 5. Relative spread versus threshold for neurons from [38] as labeled, in response to a single biphasic pulse of durations $200 \mu \mathrm{s} /$ phase (x) or 400 $\mu \mathrm{s} /$ phase (o).

\section{A. Fitting of Model Parameters to AN Statistics from Cat}

We have analyzed Javel et al.'s cat data from [38] in order to estimate the distribution of single-pulse I/O functions present in the cat AN. Stochastic model fits to 15 neurons had a mean correlation coefficient of 0.99 , with a minimum of 0.94 .

In Fig. 5, relative spread is plotted against threshold for each neuron in response to a single biphasic pulse of duration 200 $\mu \mathrm{s} / \mathrm{phase}$ or $400 \mu \mathrm{s} / \mathrm{phase}$. The RS values at a pulse duration of $200 \mu \mathrm{s} / \mathrm{phase}$ have a mean of 0.12 and a standard deviation of 0.05 , and a pulse width of $400 \mu \mathrm{s} /$ phase yields similar values ( 0.11 and 0.04 , respectively). This range of RS values is consistent with other data from the cat AN [46].

The distribution of threshold values is confounded by the fact that the distance from the stimulating electrode to the fiber, or more exactly the distance to the initial site of action potential generation in the fiber, is unknown. Van den Honert and Stypulkowski have plotted AN fiber thresholds to intracochlear electrical stimulation against cochlear place (as inferred from the AN fiber's characteristic frequency to acoustic stimulation) [47]. Since the cochlear place of the stimulating electrodes was known, it was, therefore, possible to estimate the site of each AN fiber relative to the stimulating electrode. For monopolar and longitudinal bipolar stimulation, the range of thresholds for fibers close to the stimulating electrode was on the order of $10-14 \mathrm{~dB}$. This is consistent with the range of thresholds seen in our cat data, considering the effect of distance from the stimulating electrode, and with other data from the cat AN [46].

To extend the model such that we can use it to investigate pulse widths greater than those used in collecting the Javel et al. data, the previously unpublished data collected by Dynes was analyzed, and RS was determined for neural responses to symmetric anodic/cathodic biphasic stimuli of durations up to $5000 \mu \mathrm{s} / \mathrm{phase}$.

Fig. 6 shows discharge probability versus stimulus intensity curves for three different pulse widths from Cat 76: Unit 2

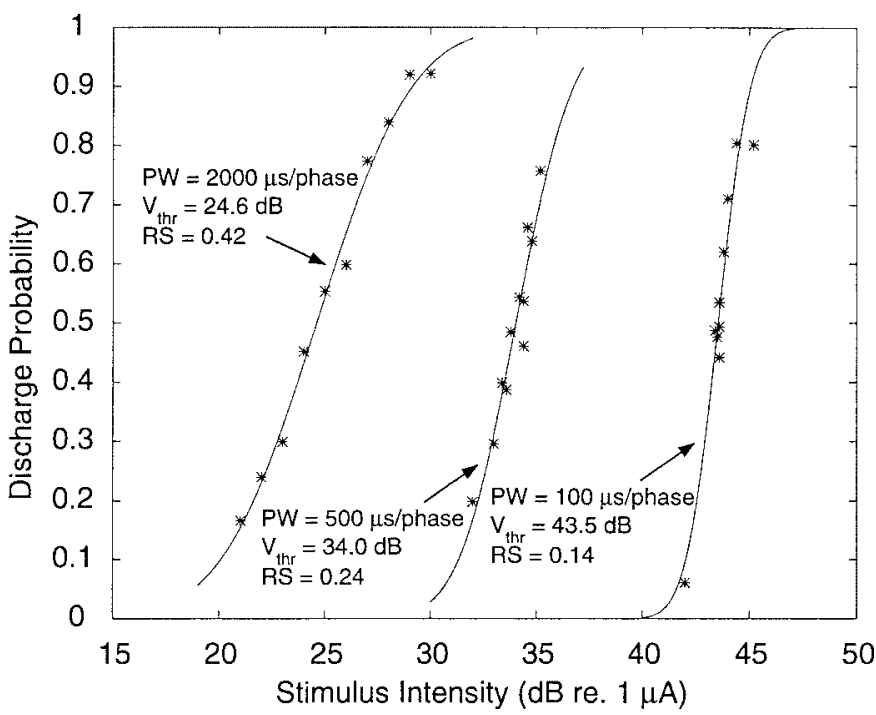

Fig. 6. Discharge probability versus stimulus intensity for single symmetric biphasic anodic/cathodic pulses of durations 100, 500, and $2000 \mu \mathrm{s} / \mathrm{phase}$ from Cat 76: Unit 2 in the Dynes data set. Plotted are individual measures (asterisks) and stochastic model fits (solid lines).

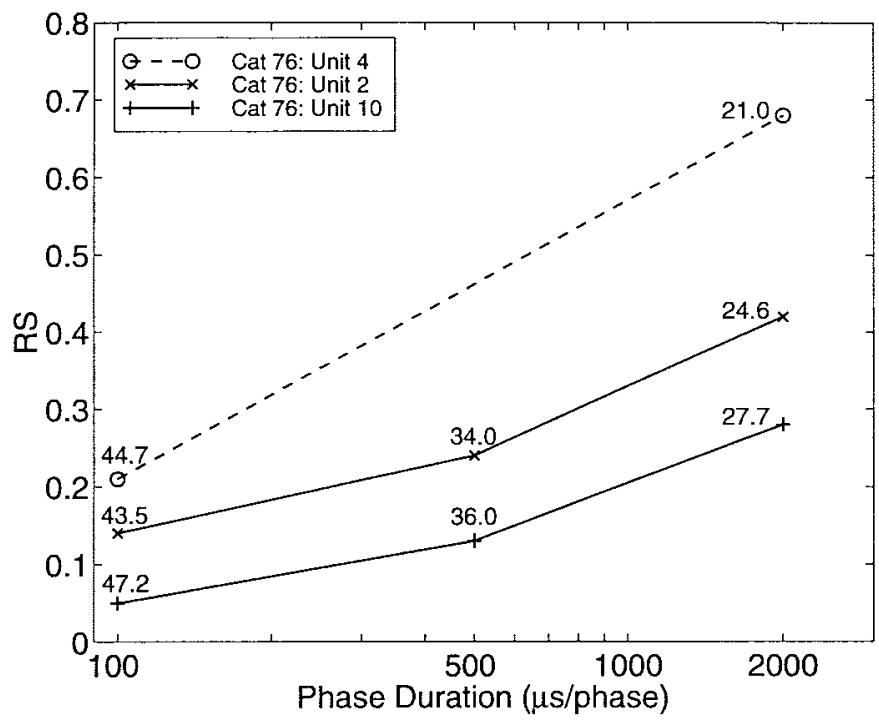

Fig. 7. Relative spread as a function of pulse width for three fibers (as listed in the legend) from the same cat in the Dynes data set. Corresponding values of threshold in $\mathrm{dB}$ re. $1 \mu \mathrm{A}$ are given for each data point.

in the Dynes data set. As the pulse width increases, the slope of the curve becomes shallower, indicating a greater dynamic range. Computing the RS from these curves shows that the RS increases as the pulse width of the anodic/cathodic biphasic stimulus increases.

Fig. 7 shows this effect in three fibers from the same cat. For every fiber, the RS increases as the duration/phase of the stimulus increases. The increase of RS with increasing pulse width as seen in Fig. 7 has also been observed in cat data from a different study [48]. Even earlier evidence of the potential importance of phase duration on auditory I/O slopes was observed in the anteroventral cochlear nucleus using sinusoidal stimuli (e.g., see [44, Fig. 33]). 


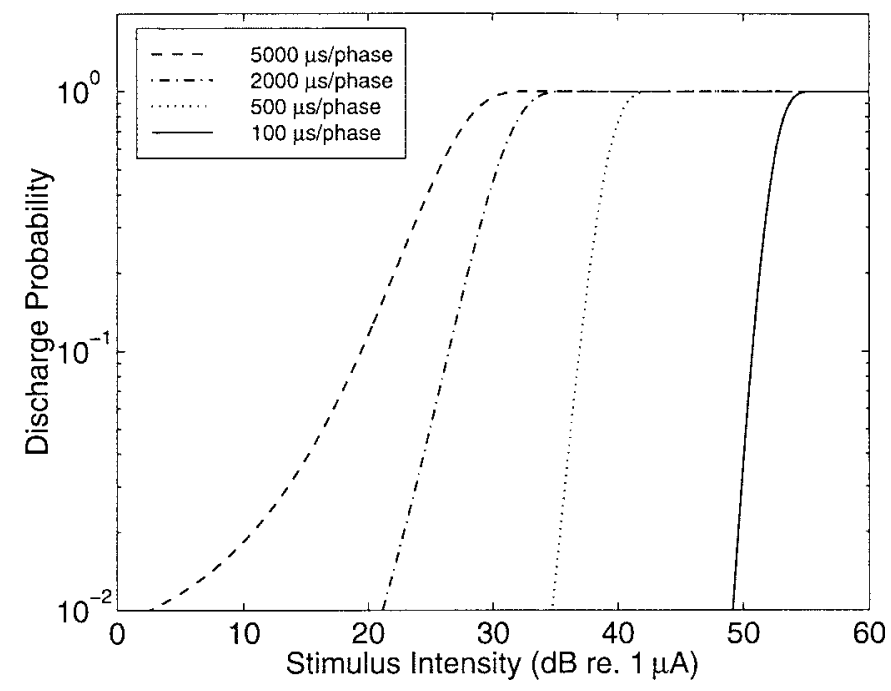

Fig. 8. Fits to discharge probability versus stimulus intensity from the Dynes data set for pulse widths of $100-5000 \mu \mathrm{s} /$ phase.

Integrated-Gaussian fits to the complete data set are shown in Fig. 8. The curves were generated from (3) using the mean values of threshold and RS at each pulse width. Even at some of the medium pulse widths, there are significant changes in the slopes of the discharge probability curves. For example, the slope changes by approximately $1.67: 1$ when the pulse width is lowered from 2000 to $500 \mu \mathrm{s} /$ phase.

There are insufficient data to examine how the distributions of threshold and RS covary with pulse width. Therefore, we assume that the distributions do not change with pulse width. It may also be feasible that the standard deviation of RS covaries linearly with its mean-further data would be required to determine this.

The available data (see Fig. 5) do not support the hypothesis that RS is a function of threshold. When more data become available, it may be possible to detect such a dependency. Meanwhile, we assume that the distribution of single-pulse $\mathrm{I} / \mathrm{O}$ functions present in the normal cat cochlea can be well described by two independent random variables, "Threshold" and "RS."

The mean single-fiber threshold and RS in a population of fibers are estimated by fitting appropriate functions to the values of threshold and RS of the curves in Fig. 8, producing the relationships

$$
E[\text { Threshold }]=121.04 \times \mathrm{PW}^{-0.18}(\mathrm{~dB} \text { re. } 1 \mu \mathrm{A})
$$

and

$$
E[\mathrm{RS}]=0.12+9.51 \times 10^{-5} \times \mathrm{PW}-7.90 \times 10^{-9} \times \mathrm{PW}^{2} \text {. }
$$

for $100 \leq \mathrm{PW} \leq 5,000$, where $\mathrm{PW}$ is the pulse width in $\mu \mathrm{s} / \mathrm{phase}$.

Note that we cannot assume that $V_{\text {noise }}$ does actually remain constant for the duration of a long pulse, as stated in the approximations listed in the first column of page 620, and that the discharge probability is well described by an integratedGaussian. Therefore, for longer pulse widths these equations form a more phenomenological description of the I/O function.
Additionally, (5) corresponds to an average chronaxy on the order of $1000 \mu \mathrm{s}$, which is somewhat longer than most other AN data and models [11]. This may be an artifact of the small amount of data used to derive (5)—more data may be required to improve its accuracy.

Taking a conservative estimate of the distribution of thresholds seen in the cat data, Threshold is taken to be uniformly distributed $\pm 5 \mathrm{~dB}$ around its mean as given by (5). From Fig. 5, the standard deviation of RS is approximately half its mean at short pulse widths (200 and $400 \mu \mathrm{s} /$ phase). Therefore, we take RS to be normally distributed with a standard deviation of 0.06 [half the mean of RS as given by (6) at short pulse widths].

In simulations using the total AN model, pseudorandom numbers with the correct distributions are generated to obtain values of threshold and RS for each fiber. In order to maintain the same set of I/O functions across simulations with the same number of fibers, the seeds of the pseudorandom number generators are two different fixed values, one for the generation of the thresholds and one for the RS's.

\section{B. Current Spread}

The two electrode configurations that we will investigate in this paper are commonly known as monopolar and bipolar. In the case of monopolar stimulation, the active electrode is one of the electrodes on the array within the cochlea and the return electrode is an electrode external to the cochlea. In the case of bipolar stimulation, both the active electrode and the return electrode are on the electrode array within the cochlea.

Following [45], we approximate the electrode array by a point source of current at the active electrode and the AN tissue by a homogeneous resistive medium consisting of a uniform density of single AN fibers. We can then calculate the potential at the site of each auditory neuron for a given stimulus intensity. From [49], the current decays at a rate of $3-6 \mathrm{~dB} / \mathrm{mm}$ for bipolar stimulation. We extend this model to also give the current spread for monopolar stimulation. The attenuation is taken to be approximately 0.5 $\mathrm{dB} / \mathrm{mm}$ for monopolar stimulation and $4 \mathrm{~dB} / \mathrm{mm}$ for bipolar stimulation - the latter value is appropriate for both radialbipolar pairs [50] and narrowly focused longitudinal-bipolar pairs [49]. Modeling an electrode placed $15 \mathrm{~mm}$ inside a cochlea $30-\mathrm{mm}$ long produces attenuation curves as shown in Fig. 9.

Note that this simple description of current spread may be modified to take into account nonsymmetrical current spread [51] and/or nonmonotonic current spread effects such as crossturn stimulation in the modiolus [12].

\section{Output of Total Auditory Nerve Model}

Here we present the output of a model of 10000 independent neurons $^{2}$ spread uniformly ${ }^{3}$ across the AN, assuming the

\footnotetext{
${ }^{2}$ This is a conservative estimate of the number of surviving AN fibers in a subject with a profound sensorineural hearing loss [52].

${ }^{3} \mathrm{~A}$ nonuniform distribution would further increase the importance of differences in single-fiber I/O functions within a local population. See [23] and [24] for a discussion of these effects.
} 


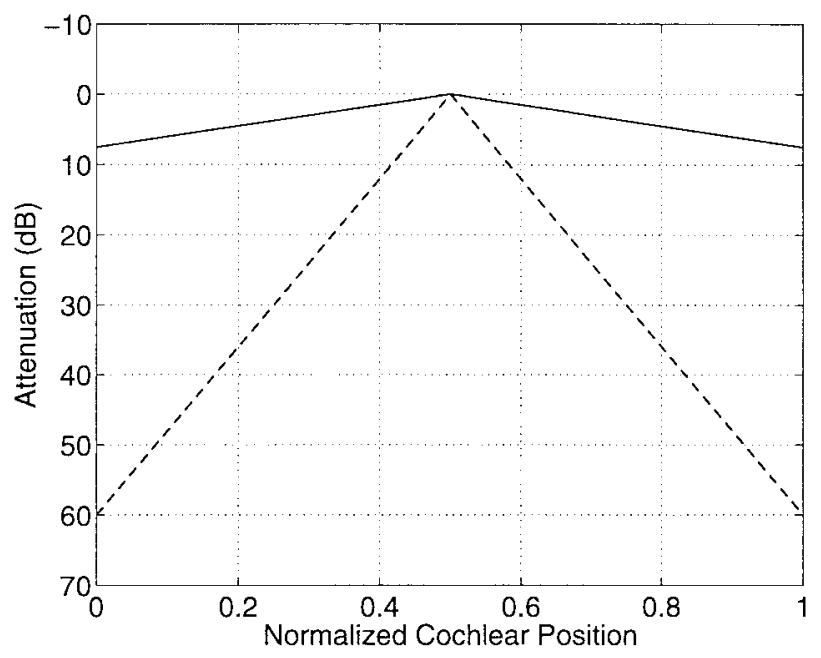

Fig. 9. Attenuation of the stimulus across the cochlea for monopolar (solid line) and bipolar (dashed line) electrode configurations.

statistics of model parameters given in Section IV-A and the model of current spread given in Fig. 9. In Fig. 10 the mean number of discharges summed across all fibers in response to a single biphasic pulse is plotted. For stimulus intensities above approximately $50 \mathrm{~dB}$, the deterministic model and the stochastic model produce very similar predictions of the mean number of discharges. However, below $50 \mathrm{~dB}$ the stochastic model predicts a quite different response from the deterministic model's. Note that the stochastic model predicts a mean number of discharges of greater than 70 spikes in response to a single biphasic pulse of intensity $50 \mathrm{~dB}$ in bipolar mode and greater than 630 spikes in monopolar mode. It is very likely that auditory threshold is reached with an even smaller number of responses, so it is highly feasible that cochlear implants are operating in the region where the stochastic activity is significant [22]-[24]. Furthermore, the deterministic model will predict zero variance in the number of discharges, whereas the stochastic model will predict nonzero variance [see error bars in Fig. 10(b)].

The summing of these Bernoulli random variables is called Poissonian binomial sampling [53], which is similar to a binomial process, except that the probability of success (discharge probability in our case) is different, in general, for each neuron. When the number of neurons is large, the Poissonian binomial distribution is well approximated by a Poisson distribution if the mean number of responses is less than 15 and by a Gaussian (Normal) distribution for mean counts greater than or equal to 15 , although the distribution will only have discrete integer values in both cases.

Fig. 11 illustrates results from 10000 iterations of Monte Carlo simulations for a very low amplitude stimulus [Fig. 11(a)] and a moderate amplitude stimulus [Fig. 11(b)], with a Poisson distribution fit to the former and a Gaussian distribution fit to the latter. The fits appear to be quite good.

\section{DISCUSSION}

We have shown that a deterministic model is a poor description of a neuron's response to single electrical pulses, in

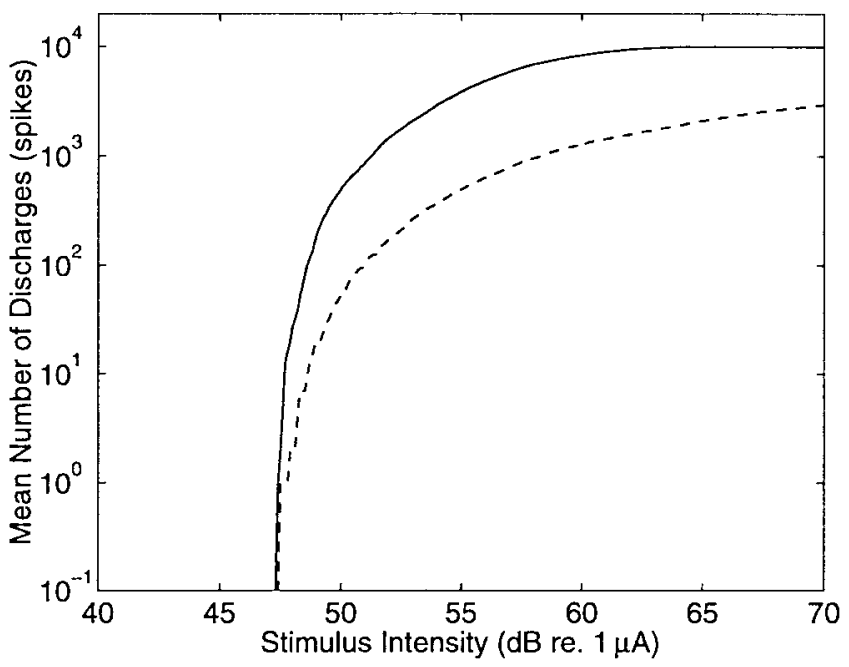

(a)

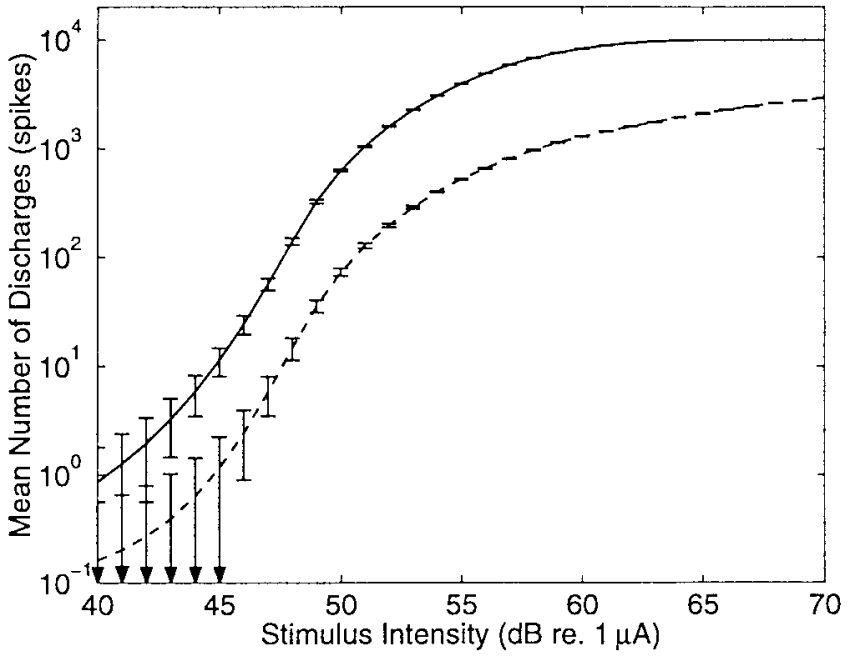

(b)

Fig. 10. Mean number of discharges versus stimulus intensity from model of 10000 fibers in response ot s single biphasic pulse for monopolar (solid line) and bipolar (dashed line) stimulation. (a) Deterministic model and (b) stochastic model. Error bars indicate +-1 std.

light of the improvements in the prediction of physiological data when a stochastic component of response is added to the neural model. Specifically, we have found that a simple stochastic model can accurately predict discharge probabilities present in cat data in response to single biphasic pulses. Our results suggest that just two parameters (threshold and RS) can well describe discharge probabilities in AN fibers in response to single biphasic pulses. As mentioned in Section I, the model methodology may also be suitable for functional electrical stimulation of other types of nerve fibers. However, use of this model for a paradigm other than stimulation of the AN by a cochlear implant would require validation of the assumptions and approximations of this paper for the particular stimulus and neural properties of that paradigm.

The analytical descriptions of single fiber response to single electrical pulses presented in this paper provide a simple and computationally efficient method of modeling the response of a large-scale population of AN fibers to pulsatile electrical 


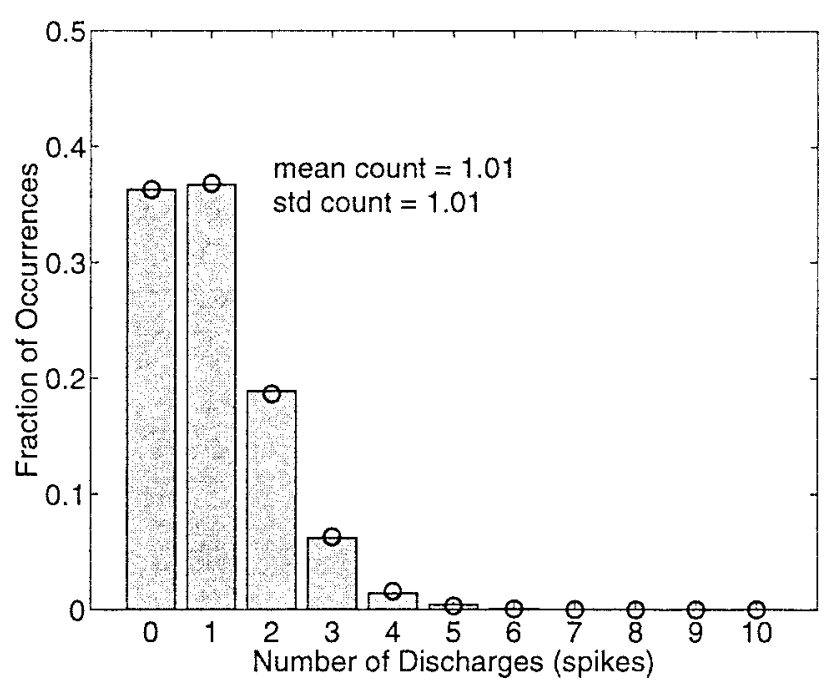

(a)

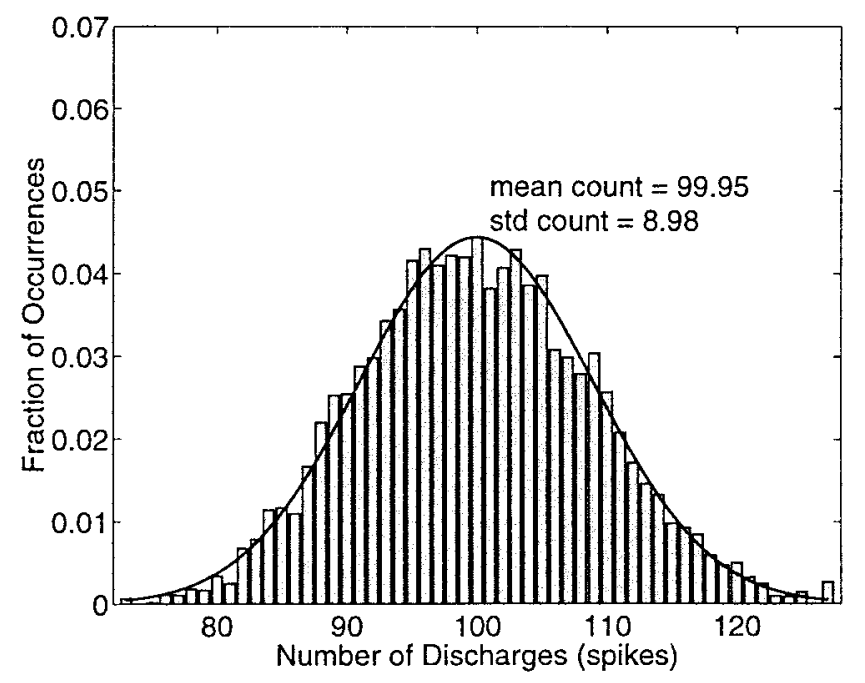

(b)

Fig. 11. Distribution of total number of discharges predicted by 10000 iterations of Monte Carlo simulations in response to a single biphasic pulse for two different intensities, with a Poisson distribution fit (o) for the case where the mean count less than 15 and a Gaussian (Normal) distribution fit (solid line) for the case where the mean count is greater than 15. (a) Low-intensity stimulus and (b) moderate-intensity stimulus.

stimulation as produced by cochlear implants. Furthermore, the output of the model permits the direct use of signal detection theory to determine the resolution with which the AN can convey information about an electrical stimulus [22]-[24].

Further physiological and modeling studies would be helpful in refining and extending a number of aspects of our model-specific suggestions follow.

In this paper, we have investigated randomness in the probability of response to electrical stimuli. Physiological studies have shown that randomness occurs not only in the probability of an action potential being generated, but also in its latency [16], [27], [38], [54], [55]. The term "jitter" is used qualitatively to describe this phenomenon, or quantitatively to refer specifically to the standard deviation of the latency [54]. In developing an analytical description of discharge probability we have degraded the model's description of the timing of the discharges. A separate stochastic description of action potential latency would be needed to investigate the effects of jitter in theories of coding that are dependent on the exact timing of discharges.

Our approximation that discharges occur only in response to the cathodic phase of the biphasic pulse (see the approximations listed in the first column on page 620) has not appeared to degrade our model's fits of the AN data. However, physiological studies have shown that some AN fibers do exhibit a shift in the phase of the biphasic current pulse to which they respond [55]. Such a phenomenon may have an effect on the I/O function of these fibers. For example, the cause of the increase in RS with increasing pulse width is as yet unknown-in this paper, we have only provided a phenomenological description of this effect. This behavior is absent in responses to monophasic pulsatile stimuli [6], [7], [9], suggesting that the effect is in fact due to the addition of the leading anodic phase in the stimulus. There are a number of ways in which the anodic phase could increase RS. One possibility is that the relative level of noise at the initial site of activation increases with pulse width. Verveen and Derksen [29] showed that the membrane noise tends to increase when the transmembrane potential deviates from its resting potential [see Fig. 1(b)]. This means that the anodic phase of the pulse could change the membrane potential and, thereby, increase the RS of a following suprathreshold cathodic phase, if the relative noise level changes more for longer pulse widths and is sustained until the presentation of the cathodic phase. A second possibility is that the initial site of activation changes with alterations in pulse width in a way that does not occur for monophasic stimuli. Different activation sites (i.e., nodes of Ranvier) in the one fiber could have different diameters and different channel densities, producing different relative spreads [5], [30]. However, the model does not take into account the spatial distribution of the stimulus potential along the nerve fiber, and it, therefore, cannot predict the node of Ranvier where the initial excitation takes place. Further physiological investigation with a combination of monophasic (cathodic and anodic) and biphasic (anodic/cathodic and cathodic/anodic) stimuli may be required to come to a proper understanding of such effects. For instance, fibers close to the passive electrode in bipolar stimulation may respond preferentially to the anodic phase and therefore, like responses to monophasic pulses, may not exhibit the same pulse-width effects as fibers responding to the cathodic phase.

The fits given in Fig. 8 were to data covering a range of discharge probabilities from approximately 0.05 to 0.95 . For biphasic pulses of short duration, Verveen and Derksen's data [29] suggest that this accuracy may extend at least as far as 0.001 to 0.999 . However, the integrated-Gaussian fit is probably a poor approximation for discharge probabilities lower than 0.05 for biphasic pulses of long duration. First, Fig. 12 shows that as the stimulus intensity goes to zero the discharge probability goes to different values for different pulse widths. This is not possible however, since the pulse width of a nonexistent (zero intensity) current pulse cannot affect the discharge probability. Second, Verveen and Derksen showed that the noise distribution departs from a Gaussian 


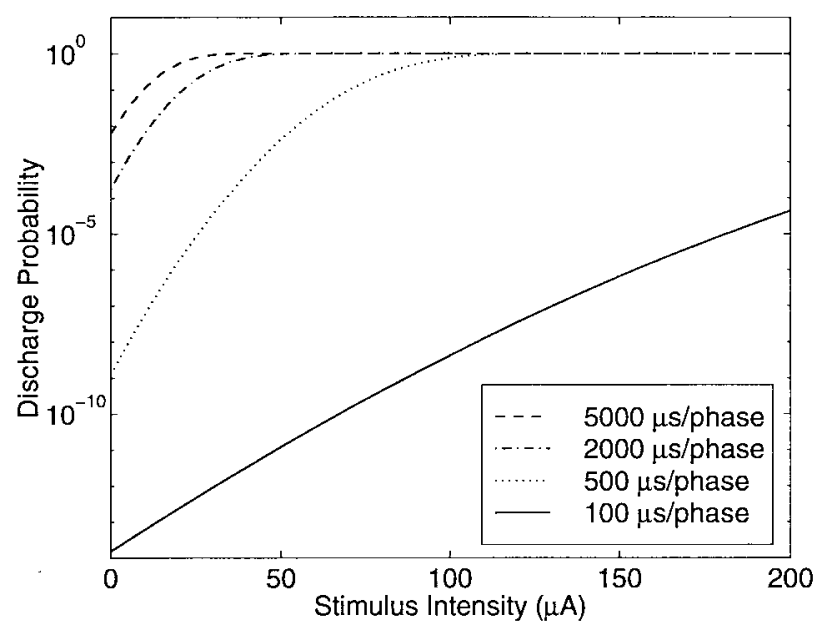

Fig. 12. Fig. 8 replotted with a linear abscissa and an extended ordinate.

(i.e., is skewed) when the membrane is hyperpolarized. If the increase in RS for longer pulse durations results from hyperpolarization of the membrane by the anodic phase of a biphasic pulse, then not only may the relative noise level be increased, but the noise distribution may also become nonGaussian. These two observations suggest that further data need to be collected to show how the discharge probability changes with pulse width for probabilities considerably smaller than 0.05 .

The small amount of data used in Section IV-A to investigate how the distributions of threshold and RS vary with stimulus parameters lead to somewhat rough approximations of their behavior. A study similar to van den Honert and Stypulkowski's [47], but in which RS values are collected along with threshold values, would be useful in further refining the statistics of our total AN model. In particular, the relationships between site of the electrode, pulse width, threshold and RS need to be further examined.

The physiological data presented in this paper and the models derived from this data were from cats. Care should be taken in extrapolating the model to other species, including human cochlear implant users. Furthermore, other than assuming zero inner hair cell survival and allowing an arbitrary number of surviving AN fibers, this model does not take into account the effects that prolonged deafness has on the response of AN fibers to electrical stimulation [56]. An extension to this study could be to model the effects of various etiologies on single-fiber I/O functions.

An application of this work concerns both the i) effectiveness and the ii) safety of high-rate pulsatile stimulation in cochlear implant stimulation strategies. Unless the stochastic nature of the AN is considered, stimulus pulse-rates much higher than an auditory fiber's maximum discharge-rate appear to be without value and very possibly dangerous to the nerve.

Nerve damage studies have found that stimulation at high pulse-rates and high amplitudes does induce serious injury to the nerve [25], [57]. However, at lower stimulus amplitudes that are within the animal's behavioral range nerve damage is not observed [26]. One plausible explanation for these results is directly related to the importance of the stochastic nature of AN. If we stimulate the nerve with a high pulse-rate, low-amplitude stimulus, we would expect that each fiber's discharge rate could be much lower than the stimulus's high pulse rate [21], [38]. As a consequence, no damage to the fibers would occur. However, if the stimulus were high in amplitude, fiber discharge rates would indeed become too high [21], [38] and cause injury to the nerve.

Until the stochastic nature of AN was considered plausible, stimulation with high-rate pulse trains for conveying finegrain temporal information [58] appeared to be nearly futile. Interestingly, the high-rate interleaved pulsatile stimulation processor was proposed [50, see page 334], ${ }^{4}$ and constructed in 1985 at the same laboratory that first introduced the stochastic nature of the AN to the cochlear implant community [3].

Several cochlear implant research groups have suggested that increasing the relative noise level of nerve fibers may improve the "temporal representation" of the stimulus. It is argued that: i) noisier fibers will reduce the occurrence of highly synchronous discharge patterns that are not seen in responses to acoustic stimulation and ii) noisier fibers will reduce refractory effects that distort the temporal representation of the stimulus. This may be a very useful strategy. Our recent work (this paper, and [21]-[24]) suggests several methods for increasing the relative noise level of fibers. However, such a strategy may be entirely unnecessary. If an implant user's nerve fibers discharge only at low probabilities during multichannel continuous stimulation, refractory effects will be negligible because discharge rates will be low. Also, abnormally high synchronization of discharges across fibers will not occur. For example, with a 1000 pulses/s stimulus and fiber discharge probabilities less than 0.1 , relatively little "temporal distortion due to refractory effects" will occur. This is because the average interval between discharges within any fiber will be greater than $10 \mathrm{~ms}$.

If the normal behavioral operating range of cochlear implants falls totally in the region above $50 \mathrm{~dB}$ in Fig. 10, then the deterministic model will adequately predict the mean number of discharges, although it will be unable to predict the variance in the number of discharges. However, if some or all of the operating range falls in the region below $50 \mathrm{~dB}$, Fig. 10 indicates that the two models predict very different responses. Physiological and behavioral studies in the same animals provide one method of determining the intensities at which the physiological data should be collected [48]. An alternative method is to model how an ideal observer would behave from the output of the AN model. We carry out such an evaluation in [23] and [24], where we predict a range of psychophysical measures using this AN model. The results of this study suggest that cochlear implants are indeed operating in the region where stochastic activity is significant. In all the cases investigated, the stochastic model predicted psychophysical performance significantly better than the deterministic model did. This suggests that the stochastic model is not just more

\footnotetext{
${ }^{4}$ Interleaved stimulation for cochlear implants was first proposed by Eddington [personal communication 1976], [59] for the purpose of eliminating interchannel interactions due to electric field summation at the electrode-nerve interface.
} 
accurate in its prediction of physiological response, but also in its prediction of the resulting behavioral performance.

\section{ACKNOWLEDGMENT}

The authors would like to thank J. Tong and R. Shepherd for their part in collecting the physiological data of [38], R. Shepherd, A. Burkitt, L. Cohen and the four anonymous reviewers for invaluable comments on the manuscript, and C. van den Honert and C. Finley for their assistance given to $\mathrm{M}$. White in initial investigations of stochastic activity in the auditory nerve.

\section{REFERENCES}

[1] L. Irlicht, D. Au, and G. Clark, "New temporal coding scheme for auditory nerve stimulation," Ann. Otol. Rhinol. Laryngol., vol. 104, no. Suppl. 166, pp. 358-360, 1995.

[2] L. S. Irlicht and G. M. Clark, "Control strategies for neurons modeled by self-exciting point processes," J. Acoust. Soc. Amer., vol. 100, no. 5, pp. 3237-3247, 1996.

[3] M. W. White, Design considerations of a prosthesis for the profoundly deaf. Ph.D. dissertation, Univ. California, Berkeley, 1978.

[4] I. J. Hochmair-Desoyer, E. S. Hochmair, H. Motz, and F. Rattay, "A model for the electrostimulation of the nervus acusticus," Neurosci. vol. 13, no. 2, pp. 553-562, 1984

[5] M. W. White, C. C. Finley, and B. S. Wilson, "Electrical stimulation model of the auditory nerve: Stochastic response characteristics," in Proc. 9th Annu. Conf. IEEE Engineering in Medicine and Biology Society, Boston, MA., 1987, pp. 1906-1907.

[6] J. T. Rubinstein, "Threshold fluctuations in an $\mathrm{N}$ sodium channel model of the node of Ranvier," Biophysical J., vol. 68, pp. 779-785, 1995.

[7] S. Dynes, Discharge characteristics of auditory nerve fibers for pulsatile electrical stimuli, Ph.D. dissertation, Massachusetts Institute of Technology, Cambridge, MA, 1996.

[8] S. J. O'Leary, L. S. Irlicht, I. C. Bruce, M. W. White, and G. M. Clark, "Variance of spike rate from auditory nerve following electrical pulsetrain stimulation of cochlea: An experimental and modeling study," $J$. Acoust. Soc. Amer., submitted for publication.

[9] H. Lecar and R. Nossal, "Theory of threshold fluctuations in nerves," Biophysical J., vol. 11, pp. 1048-1067, 1971.

[10] J. R. Clay and L. J. DeFelice, "Relationship between membrane excitability and single-channel open-close kinetics," Biophysical J., vol. 42, pp. $151-157,1983$

[11] J. Colombo and C. W. Parkins, "A model of electrical excitation of the mammalian auditory-nerve neuron," Hear. Res., vol. 31, pp. 287-312, 1987.

[12] J. H. M. Frijns, S. L. de Snoo, and R. Schoonhoven, "Potential distributions and neural excitation patterns in a rotationally symmetric model of the electrically stimulated cochlea," Hear. Res., vol. 87, pp. 170-186, 1995.

[13] A. Hodgkin and A. Huxley, "A quantitative description of membrane current and its application to conduction and excitation in nerve," $J$. Physiol., vol. 117, pp. 500-544, 1952.

[14] B. Frankenhaeuser, "A method for recording resting and action potentials in the isolated myelinated nerve fiber of the frog," J. Physiol. (Lond.), vol. 135, p. 550, 1957

[15] F. A. Dodge, "Ionic permeability changes underlying nerve excitation," in Biophysics of Physiological and Pharmacological Actions, A. M. Shanes, Ed., Pub. 69 of the Amer. Assoc. Advancement of Sci., Washington, D.C., p. 119, 1961.

[16] A. A. Verveen and H. E. Derksen, "Fluctuations in membrane potential of axons and the problem of coding," Kybernetik, vol. 2, no. 4, pp. $152-160,1965$

[17] A. A. Verveen, "On the fluctuation of threshold of the nerve fiber," in Structure and Function of the Cerebral Cortex, D. B. Tower and J. P. Schadé, Eds. Amsterdam, the Netherlands, Elsevier, 1960, pp. 282-288.

[18] A. A. Verveen, Fluctuation in excitability, Ph.D. dissertation, Univ. Amsterdam, Amsterdam, the Netherlands, 1961.

[19] M. T. Hoopen and A. A. Verveen, "Nerve-model experiments on fluctuation in excitability," Prog. Brain Res., vol. 2, pp. 8-21, 1963.

[20] S. J. O'Leary, L. S. Irlicht, I. C. Bruce, M. W. White, and G. M. Clark, "Prediction of variance in neural response to cochlear implant stimulation and its implications for perception," in Abstracts of the XVI
World Congress of Otorhinolaryngology Head and Neck Surgery, IFOS, Sydney, Australia, 1997.

[21] I. C. Bruce, L. S. Irlicht, M. W. White, S. J. O'Leary, S. Dynes, E. Javel, and G. M. Clark, "A stochastic model of the electrically stimulated auditory nerve: Pulse-train response," this issue, pp. 630-637.

[22] _ "An improved model of electrical stimulation of the auditory nerve," in Cochlear Implants. XVI World Congress of Otorhinolaryngology Head and Neck Surgery, G. M. Clark, Ed. Bologna, Italy: Monduzzi Editore, 1997, pp. 125-130.

[23] I. C. Bruce, Spatiotemporal coding of sound in the auditory nerve for cochlear implants, Ph.D. thesis, The University of Melbourne, Melbourne, Australia, 1997.

[24] I. C. Bruce, M. W. White, L. S. Irlicht, S. J. O’Leary, and G. M. Clark, "The effects of stochastic neural activity in a model predicting intensity perception with cochlear implants: Low-rate stimulation," submitted for publication.

[25] M. Tykocinski, R. K. Shepherd, and G. M. Clark, "Reduction in excitability of the auditory nerve following electrical stimulation at high stimulus rates," Hear. Res., vol. 88, pp. 124-142, 1995.

[26] J. Xu, R. K. Shepherd, R. E. Millard, and G. M. Clark, "Chronic electrical stimulation of the auditory nerve at high stimulus rates: A physiological and histopathological study," Hear. Res., vol. 105, pp. $1-29,1997$

[27] A. A. Verveen and H. E. Derksen, "Fluctuation phenomena in nerve membrane," Proc. IEEE, vol. 56, pp. 906-916, June 1968.

[28] H. E. Derksen and A. A. Verveen, "Fluctuations of resting neural membrane potential," Science, vol. 151, pp. 1388-1389, 1966.

[29] A. A. Verveen and H. E. Derksen, "Amplitude distribution of axon membrane noise voltage," Acta Physiol. Pharmacol. Neerl., vol. 15, pp. 353-379, 1969.

[30] A. A. Verveen, "Axon diameter and fluctuation in excitability," Acta Morph. Neerl.-Scand., vol. 5, pp. 79-85, 1962.

[31] H. Spoendlin and A. Schrott, "Analysis of the human auditory nerve," Hear. Res., vol. 43, pp. 25-38, 1989.

[32] O. Gleich and S. Wilson, "The diameter of guinea pig auditory nerve fibers: Distribution and correlation with spontaneous rate," Hear. Res., vol. 71, pp. 69-79, 1993.

[33] M. C. Liberman and M. E. Oliver, "Morphometry of intracellularly labeled neurons of the auditory nerve: Correlations with functional properties," J. Comp. Neurol., vol. 223, pp. 163-176, 1984

[34] A. L. Hodgkin, The Conduction of the Nervous Impulse. Liverpool, U.K.: Liverpool Univ. Press, 1961.

[35] F. J. Sigworth, "The variance of sodium current fluctuations at the node of Ranvier," J. Physiol., vol. 307, pp. 97-129, 1980

[36] A. V. Hill, "Excitation and accommodation in nerve," in Proc. R. Soc. $B, 1936$, vol. 119 , pp. $305-355$.

[37] W. M. Kistler, W. Gerstner, and J. L. van Hemmen, "Reduction of the Hodgkin-Huxley equations to a single-variable threshold model," Neural Comput., vol. 9, no. 5, pp. 1015-1045, 1997.

[38] E. Javel, Y. C. Tong, R. K. Shepherd, and G. M. Clark, "Responses of cat auditory nerve fibers to biphasic electrical current pulses," Ann. Otol. Rhinol. Laryngol., vol. 96, no. Suppl. 128, pp. 26-30, 1987.

[39] E. Javel, "Suppression of auditory nerve responses. I. Temporal analysis, intensity effects and suppression contours," J. Acoust. Soc. Amer., vol 69, no. 6, pp. 1735-1745, 1981.

[40] J. B. Ranck, Jr., "Which elements are excited in electrical stimulation of mammalian central nervous system: A review," Brain Res., vol. 98, pp. 417-440, 1975

[41] N. Y. S. Kiang, T. Watanabe, E. C. Thomas, and L. F. Clark, (1965) Discharge Patterns of Single Fibers in the Cat's Auditory Nerve, Available: Res. Monogr. nr. 35, M.I.T. Press, Cambridge, MA, 1965.

[42] N. Y. S. Kiang, E. C. Moxon, and R. A. Levine, "Auditory-Nerve activity in cats with normal and abnormal cochleas," in Sensorineural Hearing Loss, A Ciba Foundation Symposium, G. E. W. Wolstenholme and J. Knight, Eds. London, U.K.: Churchill, 1970, p. 241.

[43] J. L. Devore, Probability and Statistics for Engineering and the Sciences, 2nd ed. Monterey, CA: Brooks/Cole, 1987.

[44] M. W. White, "Psychophysical and neurophysiological considerations in the design of a cochlear prosthesis," Audiol. Ital., vol. 1, no. 2, pp. 77-117, 1984.

[45] S. J. O'Leary, G. M. Clark, and Y. C. Tong, "Model of discharge rate from auditory nerve fibers responding to electrical stimulation of the cochlea: Identification of cues for current and time-interval coding," Ann. Otol. Rhinol. Laryngol., vol. 104, no. Suppl. 166, pp. 121-123, 1995.

[46] C. A. Miller, P. J. Abbas, J. T. Rubinstein, and A. J. Matsuoka, "The neurophysiological effects of simulated auditory prosthesis stimulation," Dept. Otolaryngology—Head and Neck Surgery and Dept. Speech, 
Pathology, and Audiology, Univ. Iowa, Iowa City, IA, 3rd Quart. Progress Rep.-NO1-DC-6-2111, 1997.

[47] C. van den Honert and P. H. Stypulkowski, "Single fiber mapping of spatial excitation patterns in the electrically stimulated auditory nerve," Hear. Res., vol. 29, pp. 195-206, 1987.

[48] D. W. Smith, "Determinants of perception with a cochlear implant," Funded grant application (Dept. Health and Human Services, Public Health Service), Duke Univ., Durham, NC, 1996.

[49] S. J. O'Leary, R. C. Black, and G. M. Clark, "Current distributions in the cat cochlea," Hear. Res., vol. 18, pp. 273-281, 1985.

[50] M. M. Merzenich and M. W. White, "Cochlear implants: The interface problem," in Functional Electrical Stimulation: Applications in Neural Prostheses, F. T. Hambrecht and J. B. Reswick, Eds., vol. 3. New York: Marcel Dekker, 1977, pp. 321-340.

[51] G. Girzon, Investigation of current flow in the inner ear during electrical stimulation of intracochlear electrodes, M.S. thesis, Massachusetts Inst. Technol., Cambridge, MA, 1987.

[52] R. Hinojosa and M. Marion, "Histopathology of profound sensorineural deafness," Ann. N.Y. Acad. Sci., vol. 405, pp. 459-484, 1983.

[53] N. L. Johnson, S. Kotz, and A. W. Kemp, "Univariate discrete distributions," Wiley Series in Probability and Mathematical Statistics, 2nd ed. New York: Wiley, 1992.

[54] C. van den Honert and P. H. Stypulkowski, "Physiological properties of the electrically stimulated auditory nerve. II. Single fiber recordings," Hear. Res., vol. 14, pp. 225-243, 1984.

[55] _ "Temporal response patterns of single auditory nerve fibers elicited by periodic electrical stimuli," Hear. Res., vol. 29, pp. 207-222, 1987.

[56] R. K. Shepherd and E. Javel, "Electrical stimulation of the auditory nerve: I. Correlation of physiological responses with cochlear status," Hear. Res., vol. 108, pp. 112-144, 1997.

[57] M. Tykocinski, R. K. Shepherd, and G. M. Clark, "Acute effects of high-rate stimulation on auditory nerve function in guinea pigs," Ann. Otol. Rhinol. Laryngol., vol. 104, no. Suppl. 166, pp. 71-74, 1995.

[58] M. W. White, "Formant frequency discrimination and recognition in subjects implanted with an intracochlear stimulating electrode," Ann. N.Y. Acad. Sci., vol. 405, pp. 123-136, 1983.

[59] D. K. Eddington, W. H. Dobelle, D. E. Brackmann, M. G. Mladejovsky, and J. L. Parkin, "Auditory prostheses research with multiple channel intracochlear stimulation in man," Ann. Otol. Rhinol. Laryngol., vol. 87, no. 6 , pp. $1-39,1978$.

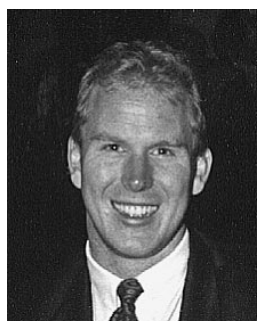

Ian C. Bruce (S'96-M'98) was born in Bendigo, Australia, in 1969. He received the B.E. (electrical and electronic) degree at The University of Melbourne, Melbourne, Australia, in 1991. In 1998, he received the Ph.D. degree from the Department of Otolaryngology, The University of Melbourne, where he developed mathematical models to investigate the coding of sound in the auditory nerve for cochlear implants.

From 1993-1994, he was a Research and Teaching Assistant in the Department of Bioelectricity and Magnetism, Vienna University of Technology, Vienna, Austria. He is presently a Postdoctoral Research Fellow in the Department of Biomedical Engineering at Johns Hopkins University, Baltimore, MD. His research interests include neural coding of sound, mathematical modeling, cochlear implants and hearing aids, neurophysiology, psychophysics, and stochastic systems.

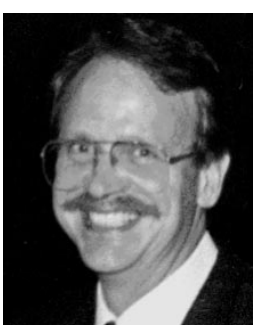

Mark W. White (M'79) received the Ph.D. degree in electrical engineering and computer science from the University of California, Berkeley, in 1978.

$\mathrm{He}$ is currently an Associate Professor in Electrical and Computer Engineering at North Carolina State University, Raleigh. His research is concerned with cochlear implants, hearing aids, biological and artificial neural networks, and machine learning.

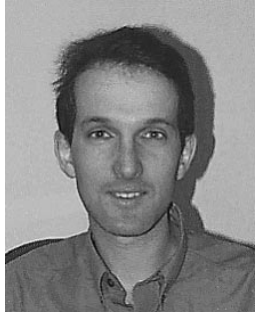

Laurence S. Irlicht (M'94) was borne in Melbourne, Australia, in 1966. He graduated with a B.Sc.(Hons.) degree in physics and the Diploma in education from The University of Melbourne, Melbourne, Australia, in 1988 and 1990, respectively. $\mathrm{He}$ received the $\mathrm{Ph} . \mathrm{D}$. degree in systems engineering from the Australian National University, Canberra, Australia, in 1993.

After an appointment as a Research Scientist at the Department of Defence in Canberra, he joined the Bionic Ear Institute, Melbourne, Australia There he applied ideas from signal processing and control theory to the related problems of determining how the brain translates neural response patterns into auditory information; and how to elicit particular neural response patterns via electrical stimulation. In 1996 he was recruited to County Investment Management, Melbourne, Australia, as a Financial Engineer. His areas of interest include information theory, system identification, signal processing, neural coding theory, control theory, and finance—and especially interrelations between these areas.

Dr. Irlicht is a member of Q-Group (Australia).

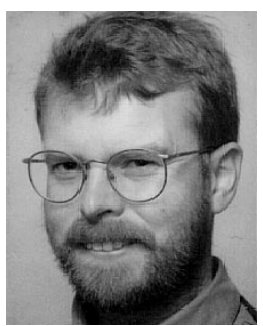

Stephen J. O'Leary was born in 1960 . He received the M.B.B.S. and B.Med.Sc. degrees from the University of Melbourne, Melbourne, Australia, in 1985. In 1994, he received the Ph.D. degree in the Department of Otolaryngology, The University of Melbourne, where he studied neural coding in the cochlear nucleus following electrical stimulation of the auditory nerve.

After completing post-doctoral research at the University Laboratory of Physiology, Oxford, he undertook surgical training in Otolaryngology-Head and Neck Surgery in the U.K. and in Sydney, Australia, and was admitted as Fellow to the Royal Australasian College of Surgeons in 1997. In 1998 he was a Clinical and Research Fellow at the University Hospital Utrecht, Utrecht, The Netherlands, and he is now a Senior Lecturer and Surgeon with the Bionic Ear Institute, Melbourne, Australia. His research interests include neural coding for cochlear implants, developmental auditory neurobiology, and inner ear biology.

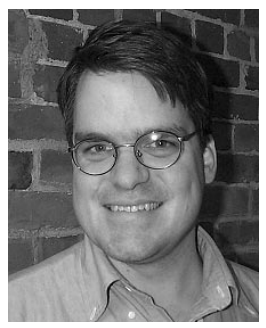

Scott Dynes was born in Seattle, WA, in $1960 . \mathrm{He}$ received the A.B. degree in physics from Princeton University, Princeton, NJ, in 1983. Later, he continued the study of physics at Massachusetts Intitute of Technology, Cambridge, first in radio astronomy and then in auditory physiology at the Eaton-Peabody Laboratory. He received the Ph.D degree in 1996 for experimental and modeling studies of auditory nerve fiber response to complex electrical stimuli.

His research interests include neural stimulation and computer-based knowledge representations.

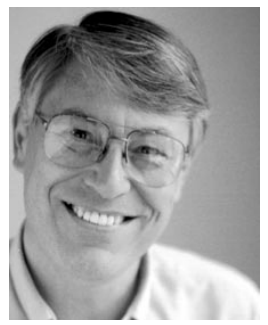

Eric Javel was born in Elizabeth, NJ, in 1947. He received the B.A. degree in biology from Johns Hopkins University, Baltimore, MD, in 1968 and the Ph.D. degree in bioacoustics from the University of Pittsburgh, Pittsburgh, PA, in 1972.

Following a postdoctoral fellowship in Neurophysiology at the University of Wisconsin-Madison, he held positions at the Boys Town National Research Hospital, Omaha, NE, where he headed the Auditory Physiology Laboratory and Center for Hearing Research, and at Duke University, Durham, NC. He is currently Associate Professor of Otolaryngology, Neuroscience and Communication Disorders at the University of Minnesota, Minneapolis. His research focuses on auditory stimulus coding, implantable auditory prostheses, and physiological bases of auditory perception. 


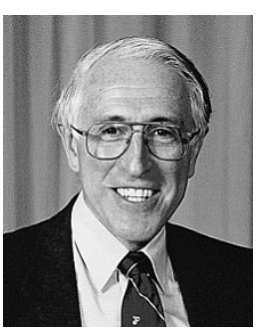

Graeme M. Clark received the M.B.B.S. degree from The University of Sydney, Sydney, Australia, in 1957. In 1967 he commenced basic research to investigate whether a single or multiple-channel (electrode) cochlear implant would be possible for the management of a profound hearing loss. He received the Master of Surgery and Ph.D. degrees from The University of Sydney, in 1968 and 1969, respectively.

$\mathrm{He}$ is foundation Professor of the Department of Otolaryngology, The University of Melbourne, Melbourne, Australia, and foundation Director of The Bionic Ear Institute. Since 1970, he has led the research team that developed the Australian cochlear implant manufactured by Cochlear Limited, Sydney, Australia, that now provides hearing to over 20000 profoundly or totally deaf children and adults.

Dr. Clark was awarded the Order of Australia in 1983 for his contribution to Medicine and in 1998 became a Fellow of the Australian Academy of Science and the Australian Academy of Technical Sciences and Engineering. 


\section{University Library}

\section{- M M N E R VA A gateway to Melbourne's research publications}

Minerva Access is the Institutional Repository of The University of Melbourne

Author/s:

Bruce, Ian C.;White, Mark W.;Irlicht, Laurence S.;O'Leary, Stephen J.;Dynes, Scott;Javel, Eric;Clark, Graeme M.

Title:

A stochastic model of the electrically stimulated auditory nerve: single-pulse response

Date:

1999

\section{Citation:}

Bruce, I. C., White, M. W., Irlicht, L. S., O'Leary, S. J., Dynes, S., Javel, E., et al. (1999). A stochastic model of the electrically stimulated auditory nerve: single-pulse response. IEEE Transactions on Biomedical Engineering, June, 26(6), 617-629.

Persistent Link:

http://hdl.handle.net/11343/27515 